\title{
AUTOCUIDADO E CUIDADO DE PESSOAS COM CÂNCER E DISPOSITIVOS MÉDICOS DE ALIMENTAÇÃO
}

SELF CARE AND CARE OF PEOPLE WITH CANCER AND USING MEDICAL FEEDING DEVICES

\begin{abstract}
JULIANA RODRIGUES FREITAS OLIVEIRA 1, MARIANA APARECIDA PEREIRA COUTINHO 2, MURILO CÉSAR DO NASCIMENTO 3, ELIZA MARIA REZENDE DÁZIO 4, FÁBIO DE SOUZA TERRA 5,
\end{abstract} MARINA BAVARESCO 6 , SILVANA MARIA COELHO LEITE FAVA 7

1., 2., 3., 4., 5., 6., 7. UNIVERSIDADE FEDERAL DE ALFENAS (UNIFAL-MG), Alfenas, Minas Gerais, Brasil

\begin{abstract}
INTRODUCTION: Cancer and its treatment can affect the ability to feed, which require enteral nutritional therapy, requires appropriate self-care and care. Objective: to know the self-care/care of people with cancer using medical feeding devices in a city of the state of Minas Gerais. METHOD: qualitative investigation obtained by means of interviews recorded, using a semi-structured instrument, with people assisted in a reference center in oncology. RESULTS: built the central theme Requirements for self-care/universal care for health deviation: propelling and restraining forces and as sub-themes facilitators for self-care engagement and complicators for self-care engagement/care. CONCLUSIONS: the results contribute to the praxis of health professionals, especially nurses, to rethink the relevance of the guidelines in the process of transition from hospital to home and to recognize that each person has a differentiated of learning and all patients have the potential for care/self-care.
\end{abstract}

KEY WORDS: Self-care, Enteral nutrition, Oncology, Nursing.

\section{CÓMO CITAR / HOW TO CITE}

Freitas Oliveira, J. R., Pereira Coutinho M. A., Do Nascimento, M. C., Eliza Rezende Dázio, M., De Souza Terra, F., Bavaresco, M., \& Coelho Leite Fava, S. M. (2020). Autocuidado e cuidado de pessoas com câncer e dispositivos médicos de alimentação. Salud \& Sociedad, 11, e3650. doi: 10.22199/issn.07187475-2020-0001

\footnotetext{
l. E-mail:juurfo@gmail.com•ORCID:https.//orcid.org/0000-0002-7808-8837;2.E-mail:mariaanacoutinho@gmail.com•0RCID: https://orcid.org/0000-0002-3942-499X;

3. murilo@unifal-mg.edu.br•ORCID: https://orcid.org/0000-0002-3436-2654; 4. E-mail: eliza.dazio@unifal-mg.edu.br•ORCID: https://0rcid.org/0000-0001-9216-6283;

5. E-mail: fabio.terra@unifal-mg.edu.br•ORCID: https://orcid.org/0000-0001-8322-3039; 6. E-mail: marinabavaresco@hotmail.com•0RCID: https://orcid.org/0000-0003-04668633; 7. E-mail: silvanaleitefava@gmail.com•0RCID: https://orcid.org/0000-0003-3186-9596
} 
O câncer é a principal causa de morbilidade e mortalidade no mundo. A Agência Internacional de Pesquisa em Câncer (IARC), que faz parte da OMS, apontou que 14 milhões de novos casos foram notificados no ano de 2012, sendo cerca de $60 \%$ na África, Ásia, América Central e do Sul (Nascimento et al., 2018). Estima-se cerca de 600 mil casos novos registrados no Brasil por ano nos anos 2018-2019, sendo em sua maioria em estágio avançado da doença (INCA, 2018).

O câncer é uma doença de origem multifatorial, causado pelo crescimento e a disseminação desordenada das células. Suas manifestações clínicas na maioria das vezes apresentam sem especificidade. 0 tratamento inclui quimioterapia, radioterapia ou cirurgia, dependendo do quadro clínico, ou uma combinação destes, e a sua eficiência deriva do diagnóstico e do tratamento precoce (Oliveira, Reis et al., 2018).

Esses tratamentos geralmente produzem efeitos colaterais, dentre eles, náuseas, alteração de peso, anorexia, disfagia e dor. Esses efeitos têm impacto nos aspectos físicos e emocionais, sociais, na mudança de rotina e nas relações familiares. As pessoas com câncer do trato gastrointestinal, podem apresentar como efeitos da terapia, a diminuição do apetite, a disfagia e a má nutrição, e como consequência, a desnutrição (Oliveira, Reis et al. 2018; Mitchell et al. 2017).

Quanto ao câncer de cabeça e pescoço, os efeitos mais comuns da radioterapia, tratamento preconizado de escolha, são a xerostomia e a mucosite, que podem levar a disfagia e, consequentemente, a mudança alimentar, conduzindo à perda de peso, anorexia, caquexia e desidratação, sendo necessária uma rígida avaliação do estado nutricional (Primo et al., 2016).

Percebe-se que a desnutrição é uma consequência comum associada ao câncer e aos efeitos do tratamento, e a sua presença tende a diminuir 0 efeito das terapias, aumentar sua toxicidade, além de contribuir para a debilidade do estado de saúde. Uma das maneiras de minimizar a desnutrição dessas pessoas em tratamento, que não conseguem se alimentar por via oral, é a terapia nutricional enteral (TNE) (Mitchell et al., 2017).

A TNE é considerada uma terapêutica importante, realizada por meio da inserção do dispositivo, seja por sonda nasoentérica, gastrostomia ou jejunostomia e tem por finalidade a administração de alimentos e de medicamentos. Essa terapia demanda cuidados específicos para a administração correta de alimentos e/ou medicamentos, com vistas a evitar a sua obstrução, a remoção inadvertida e a diminuição da eficácia dos fármacos.

Desse modo, para a administração de medicamentos recomenda-se a trituração dos comprimidos ou abertura de cápsulas e a dissolução em água e deve ser oferecido um por vez. Deve-se dar preferência a apresentação do fármaco na sua forma líquida e ainda, alguns medicamentos que têm a sua apresentação na forma de cápsulas não podem ser abertos, porque não diluem ou perde-se a sua eficácia. 0 não cumprimento desses princípios pode incorrer na obstrução do tubo, na diminuição da sua eficácia e na intensificação da toxicidade dos fármacos. $E$ ainda, é necessário se ter uma análise farmacológica e farmacotécnica, uma vez que as propriedades farmacológicas podem perder sua garantia (Gama et al., 2019; Joos et al., 2016).

Cabe acrescentar a importância da irrigação da sonda com água filtrada antes e após a administração da dieta e dos medicamentos. Cuidados com a fixação do dispositivo é fundamental para evitar a saída inadvertida e dobraduras na sonda (Souza et al., 2018).

Ademais, a TNE pode provocar desconforto físico, modificar a autoimagem e as atividades cotidianas, o que deve ser 
considerado no planejamento das orientações individualizadas, de modo a favorecer 0 autocuidado. Desenvolver 0 autocuidado e contar com a compreensão e 0 apoio de outras pessoas tornam 0 enfrentamento dessas experiências mais eficazes (Cai et al., 2018).

$O$ autocuidado pode ser entendido como ações executadas pela própria pessoa para a manutenção da vida, da saúde e do bemestar, a partir de condições fisiológicas, desenvolvimentais ou comportamentais, no qual não é inato, aprende-se com 0 crescimento e desenvolvimento através das relações interpessoais e da comunicação (Fialho et al., 2002; Rueda, 1999). Nesse entendimento, o autocuidado é a capacidade da pessoa em ter autonomia para tomar decisões dos caminhos a seguir sobre sua saúde e enfermidade, viabilizando a promoção, a prevenção e os cuidados diante do processo de saúde e doença (Tossin et al., 2016).

A educação em saúde, uma construção coletiva fundamentada no trabalho multiprofissional, tem por finalidade 0 estímulo ao autocuidado, porque proporciona à pessoa o empoderamento, expandindo sua autonomia e cidadania. A equipe multiprofissional desempenha um papel significativo nas práticas de educação em saúde na medida que valoriza o saber do outro, promove maior aproximação dos usuários aos serviços de saúde, cria-se espaços de partilha de diferentes saberes, 0 que pode estimular mudanças comportamentais (Barreto et al., 2019).

0 profissional de enfermagem tem um papel singular nas práticas educativas em saúde, por ser considerado o facilitador e 0 articulador das ações pela sua proximidade com os usuários. Ele atua na promoção, prevenção, tratamento e reabilitação, assistindo as pessoas de maneira pluridimensional, sendo elas biológicas, psicológicas, espirituais e socioeconômicas (Tossin et al., 2016).
As ações do enfermeiro devem contribuir para estimular à pessoa em uso da TNE e a sua família, a adesão ao autocuidado/ cuidado, a partir de informações simplistas e claras acerca da prescrição dietética, armazenamento, preparo, administração da dieta e da hidratação, além de ser o agente avaliador desse processo educativo (Cheever \& Hinkle, 2015).

O presente estudo tem o intuito de servir como um apoio para identificar as fragilidades ou potencialidades para 0 autocuidado/cuidado da pessoa em uso do dispositivo médico de alimentação, no seu cotidiano domiciliar. E permite que novos conhecimentos e habilidades sejam desenvolvidos pelas pessoas para melhor adaptação à TNE. O objetivo deste estudo foi conhecer 0 autocuidado e cuidado de pessoas com câncer em uso de dispositivos médicos de alimentação de um município da Região Sul do Estado de Minas Gerais.

\section{MÉTODO}

Trata-se de um estudo com abordagem qualitativa. Os participantes foram compreendidos por pessoas atendidas em uma instituição de referência em oncologia de um município da Região Sul do Estado de Minas Gerais. Por meio do banco de dados cedido ao grupo de pesquisa processo saúde doença na perspectiva sociocultural, levantou-se os possíveis participantes a partir do Código Internacional de Doenças (CID) relacionadas às neoplasias que acometem cabeça e pescoço, pulmão, trato gastrointestinal e pâncreas.

Adotou-se como critérios de inclusão: pessoas com idade igual ou superior a 18 anos; de ambos os sexos; atendidos no serviço de referência em oncologia com diagnóstico de neoplasia maligna que estão em uso ou que utilizaram nos últimos 30 dias, a partir da data da entrevista, dispositivos médicos de alimentação, incluindo, sondas nasogástricas, nasoentéricas, gastrostomias, jejunostomias, residentes em município de um raio de $40 \mathrm{Km}$ do centro de 
referência. Adotou-se esse critério devido ao custo e ao deslocamento. E como critérios de exclusão: pessoas que residiam em área rural.

A partir dos dados obtidos, foi realizado 0 contato com o possível participante por meio telefônico para identificar o uso de algum dispositivo para alimentação e estabeleceuse no máximo três tentativas por número. Tendo em vista o número reduzido de participantes, optou-se também por levantar in situ, ou seja, no ambulatório da oncologia do hospital de referência, sendo contatados por meio da técnica da bola de neve. Os participantes foram contatados por meio telefônico, sendo apresentados previamente os objetivos da pesquisa e posteriormente foram convidados a participar do estudo. Após o aceite por via telefônica foram agendadas visitas domiciliárias, no qual receberam orientações sobre 0 estudo e obtida a assinatura do Termo de Consentimento Livre e Esclarecido, para 0 início da coleta de dados.

Este estudo foi aprovado pelo Comitê de Ética em Pesquisa da Universidade Federal de Alfenas, UNIFAL-MG, sob parecer de $n^{\circ}$ 2.816.020 e CAAE 94986418.4.0000.5142. A identidade do participante foi preservada $\mathrm{e}$ os nomes pessoais foram substituídos por nomes fictícios à escolha do pesquisador.

A coleta de dados foi utilizada no domicílio do participante com agendamento prévio, com duração de 30-40 minutos e utilizada a entrevista semiestruturada, por meio de um instrumento constituído por duas partes. A primeira teve a finalidade de levantar as características sociodemográficas e clínicas dos participantes e a segunda, com as seguintes perguntas norteadoras: 1. Nos diga um pouco sobre suas rotinas diárias. Como passa os seus dias? 2. Quais são os cuidados com si mesmo? 3. O que mudou na sua rotina diária a partir do momento que passou a usar 0 dispositivo de alimentação? 4. Como é realizado o cuidado com esse dispositivo? 5 .
Quais são as suas facilidades e as dificuldades no manejo com o dispositivo?

Os dados foram registrados em gravador e foi imediatamente transcrito na íntegra pelos pesquisadores, preservando os vícios de linguagem. As entrevistas com os participantes aconteceram em seus próprios domicílios, em datas e horários previamente agendados, a entrevista não excedeu 0 tempo de 30-40 minutos.

Para a organização dos dados, adotou-se - referencial metodológico a Análise Temática (Braun \& Clarke, 2006), o que possibilitou identificar, analisar e relatar os padrões dos dados coletados, por meio de uma descrição detalhada dos mesmos e construir os temas e subtemas. Os dados foram analisados à luz da Teoria do Autocuidado de Dorothea Orem (Foster \& Bennett, 2000).

A Teoria do Autocuidado engloba 0 autocuidado, a ação do autocuidado, os fatores condicionantes básicos e a demanda terapêutica de autocuidado. $O$ autocuidado é a capacidade que as pessoas desempenham por si próprias ou que alguém a execute por elas para preservar a vida, a saúde, 0 desenvolvimento e o bem-estar (Foster \& Bennett, 2000).

Um conceito adicional à Teoria do autocuidado são os requisitos do autocuidado divididos em: requisitos universais, de desenvolvimento e de desvio de saúde. Os requisitos universais estão associados com os processos da vida, com a manutenção da estrutura e do funcionamento do ser humano, como suprimento adequado de água e de alimentos, o repouso, a interação social. Os requisitos de desenvolvimento representam os estágios do ciclo vital que incluem a adaptação ao novo trabalho ou a adaptação as modificações do corpo como as rugas. 0 requisito desvio de saúde está relacionado a condições de lesões ou doenças como aprender a viver com os efeitos de condições 
ou estados patológicos e com as consequências do tratamento no estilo de vida. A Teoria de autocuidado e os requisitos universais e de desvio de saúde aplicam-se ao cotidiano de vida das pessoas com os dispositivos de alimentação (Foster \& Bennett, 200).

\section{RESULTADOS}

Dos 31 possíveis participantes, nove $(29,03 \%)$ os endereços encontravam-se fora do critério de inclusão, cinco $(16,12 \%)$ negaram sua participação; quatro $(12,9 \%)$ não foram encontrados nos endereços e telefone disponibilizados; dois $(6,45 \%)$ retiraram o dispositivo em um tempo superior há 30 dias; dois $(6,45 \%)$ foram à óbito. Fizeram parte desse estudo nove participantes.

Constatou-se que a maioria dos participantes era do sexo masculino seis $(66,7 \%)$, com uma idade média de 58,7 anos, seis $(66,7 \%)$ eram católicos, com renda familiar média de $\mathrm{R} \$ 1.848,90$. Quanto à escolaridade um $(11,2 \%)$ estudou até $07^{\circ}$ ano do ensino fundamental, sete $(77,8 \%)$ estudaram entre $01^{\circ}$ ano ao $4^{\circ}$ ano do ensino fundamental, e um $(11,2 \%)$ não sabia informar o nível de escolaridade.

Em relação as comorbidades associadas, três $(33,4 \%)$ autoreferiram hipertensão arterial sistêmica, um $(11,2 \%)$ alegou depressão, gastrite nervosa e acidente vascular encefálico. Quanto aos hábitos de vida, cinco $(55,6 \%)$ alegaram ser ex tabagistas e dois $(22,3 \%)$ faziam uso do tabaco, quatro $(44,5 \%)$ já fizeram consumo de bebidas alcoólicas.

Dos nove participantes, sete $(77,8 \%)$ estavam em uso de sonda nasoentérica, com um tempo médio de utilização do dispositivo de 315,14 dias, um $(11,2 \%)$ havia retirado 0 dispositivo em um tempo inferior a 30 dias, um $(11,2 \%)$ estava em uso de gastrostomia com um tempo de 14 dias e um $(11,2 \%)$ em uso de jejunostomia, com uma permanência de 123 dias. A maioria 88,9\%, possuía câncer localizado na região cervical, sendo estes na laringe, no esôfago, próximo à tireóide, embaixo da língua, embora nenhum dos participantes soube relatar com precisão o local do câncer.

Em relação aos dados qualitativos apreende-se a presença de forças propulsoras e restritivas para o cuidado e autocuidado, sendo as forças propulsoras consideradas como facilitadoras, ou seja, que potencializaram 0 autocuidado, e as forças restritivas, ou dificultadoras, consideradas barreiras para 0 autocuidado/cuidado. Da análise dos dados construiu-se 0 tema central intitulado: Requisitos de autocuidado/cuidado universal por desvio de saúde: forças propulsoras e restritivas. Como subtemas Facilitadores para o engajamento ao autocuidado/cuidado e Dificultadores para o engajamento ao autocuidado/cuidado, conforme a Figura 1.

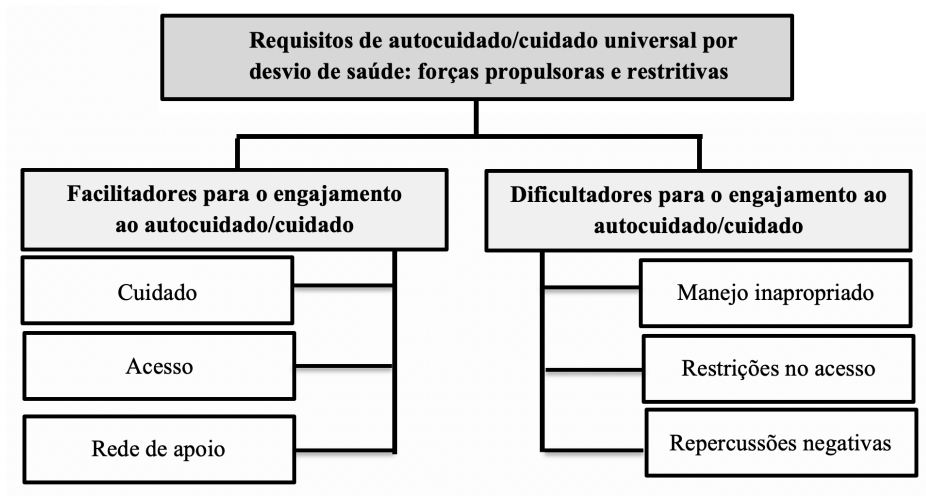

FIGURA 1.

Mapa temático com tema central e subtemas. 
Os facilitadores para o engajamento ao autocuidado/cuidado com o dispositivo de alimentação foram subcategorizados em Cuidado, Acesso e Redes de apoio.

\section{Cuidado}

O cuidado envolveu as rotinas em suas atividades diárias que permitiram com que a pessoa ou sua família não apenas se adaptasse ao uso do dispositivo, como também vislumbrasse a perspectiva da sua retirada. O ritual do autocuidado/cuidado com a dieta e prevenção de lesão é retratado nos depoimentos abaixo:

"Eu coloco no frasquinho né, aquece e ponho no banho maria, que aí não pode ser nem quente, nem frio, nem gelado [...] armazena na geladeira [...] tem que passar bem devagarinho pra não dar diarreia né [...]macetar bem macetado, dissolver ele na água pra não ta entupindo a sonda, lava também [...]" Ester

"Só muda de posição, um pouquinho para cima, um pouquinho para baixo, para não machucar (fixação) [...]" Joana

Acesso

Constatou-se que para a efetivação do autocuidado/cuidado com os dispositivos, os participantes buscavam a sua rede social que envolviam o sistema público, as Organizações não governamentais - ONGs e a famíla para o acompanhamento e para a garantia dos insumos.

"[... a cama e o suporte o padrinho que emprestou, o colchão foi pela ONG, a escadinha consegui na secretaria de saúde, o óleo de girassol foi ganhado... é complicado né, por causa do dinheiro do empréstimo, aí tem que ficar comprando essas coisas [...] 0 vida viva deu um monte, mas não deu certo de conectar (equipo) [...]" Ester

"[...] suporte de soro eu peguei emprestado, os outros materiais como seringa, frasco, eu consegui tudo pela prefeitura [...] eu tenho cadeira de rodas, cadeira de banho, muleta, muleta de braço, andador, eu consegui tudo, o leite também, remédio[...]" Cláudio

\section{Rede Social}

Entende-se por rede social o conjunto das relações que uma pessoa possui e são percebidas por ela, definindo sua comunidade social própria que the proporciona apoio em suas diferentes necessidades (Castel, 1995). 0 apoio social é a ajuda que auxilia a pessoa a ter uma melhor resposta diante dos acontecimentos da vida (Cassel, 1976).

A família e o círculo social próximo à pessoa constitui-se uma importante rede social, tendo em vista que auxiliou na realização de atividades, por vezes difíceis de serem executadas pelo participante, como também o ponto de fortaleza nos momentos difíceis da vida. O sofrimento contínuo e a possibilidade da finitude próxima do ente querido, com câncer leva a família a reaproximar e a viver bem (Sales \& Molina, 2004).Destaca-se a relevância do suporte dos profissionais de saúde ao desenvolver ações de educação em saúde no processo de transição hospital domicílio e ao estimular o processo de readaptação.

"Os filhos antes quase não conversava, voltaram a ligar, perguntar, toda hora, o dia inteiro... e acho que não ia ter aguentado não, se minhas filhas não tivessem me trazido, eu tava desanimado com a dor, eu não ia atrás não [...] tudo é as meninas, elas aprenderam a mexer sim [...]" Nelson 
"[...] todo mundo que gosta de mim me põe pra cima. O que não me faz desanimar é a minha família [...]" Carla

"Semana passada a nutricionista passou outro tipo de suplemento pra mim, mais forte, mais calórico... pediu pra mim não ficar limpando a casa, andando, falando, pra não perder as calorias [...] mas se eu começar a perder peso eu tenho que botar de novo (a sonda) [...]" Carla

Parece que a doença suscitou a fé dos participantes, constituindo uma força propulsora para o autocuidado/ cuidado, uma vez que propiciou aos participantes a ressignificação da vida, forças e esperança para superar as adversidades da doença e do tratamento.

"Deus foi abrindo as portas e preparando tudo [...] mas aí eu falo pra Deus, ai Deus eu já fui tão longe, você já me trouxe até aqui, não deixa eu desanimar não! " Carla

"[...] eu graças a Deus [...] não vai ser preciso operar não [...] O dia que eu rancar essa sonda é pra eu ir lá em Nossa Senhora Aparecida e eu vou lá se Deus quiser! "Bruno

Por outro lado, percebeu-se que algumas condições, intrínsicas ou extrínsicas dificultaram 0 autocuidado/cuidado, como 0 manejo inapropriado, as restrições no acesso e as repercussões negativas, que nesse entendimento foram consideradas como forças restritivas.

\section{Manejo Inapropriado}

Caracterizado pelo déficit do autocuidado/cuidado e contribui para prolongar o período de tratamento e para 0 aparecimento de complicações. Percebeu-se a influência negativa dos familiares e dos amigos neste processo, com ações ou orientações que contrapõem a literatura relacionada à temática.
"[...] aí nós começou a fazer comida, batia e colocava no liquidificador pra passar na sonda. É que as pessoas falavam que fazia né, a gente tava achando que era o bem, a gente cozinhava o fígado de vaca, batia no liquidificador, coava num paninho, dava e ela comia, falava que era gostoso. Ela entupiu (a sonda) três vezes por causa do alimento que colocava [...]" Carla

"No começo eu passava remédio por aqui (pela sonda), e até uma sopa ralinha de fubá, pura água sabe, bem ralinha [...]" Bruno

"[...] tinha que deixar o remédio de molho um tempo pra tomar, pra depois passar na seringa, porque a primeira prescrição que saiu era tudo comprimido [...]" Carla

"[...] quando toma amitriptilina a gente maceta junto, os dois remédios que estão no mesmo horário pode por junto [...]" Ester

Percebeu-se que os participantes apresentavam dificuldades relacionadas ao gotejamento da alimentação, a troca periódica dos insumos, a proteção da estomia durante 0 banho e as medidas corretivas diante de intercorrências.

"[...] eu vou lavando até quando às vezes ela arrebenta ou não presta (frasco e equipo), aí eu jogo fora e pego outro equipo e frasco [...] tem esse leite aqui e aí mistura com o leite de vaca [...] faz meio a meio [...] já entupiu muitas vezes [...] acontece de sair também, mas ele mesmo coloca de volta [...]" Sebastião

"Ela já deu de sair, teve um dia que eu tava tomando banho e fui rancar isso (aponta para a fixação) [...] aí eu mesmo coloquei ela." Francisco 
Restrições no Acesso

As dificuldades de acesso aos serviços, aos insumos, aos medicamentos, aos recursos materiais com qualidade, às informações e ao apoio da equipe de saúde, que são direitos da pessoa, geraram influências negativas no autocuidado/cuidado.

"Quando a sonda sai, eu mesmo coloco, porque senão tem que ficar indo toda vez lá pra Alfenas, aí tem que pedir o carro para a prefeitura [...] a maioria tem que comprar como esparadrapo, micropore... já que a prefeitura as vezes não tem a alimentação a gente mistura, são 25L para três meses [...]" Sebastião

"[...] o suplemento que ela me deu era de banana, era ruim demais, eu vomitava mesmo [...] aquele trem tem um cheiro horroroso, trem fedido, hoje mesmo eu tomo porque preciso." Carla

"[...] nunca entupiu, ele já tirou duas vezes, aí ela chamou um amigo dela que é enfermeiro e ele cobrou uma nota pra vir colocar, cobrou 120,00, colocou aqui em casa mesmo, não fez raio $x$ nem nada [...]" Cláudio

\section{Repercussões Negativas}

Percebeu-se que as adversidades da doença e os efeitos do tratamento produziram repercussões negativas com comprometimento da pluridimensionalidade da pessoa. A percepção de invalidez, de isolamento, da perda da autoestima e da identidade pode comprometer 0 autocuidado/cuidado.

"[...] tem dias que eu não consigo nem pentear o meu cabelo, pegar uma caneta, ou um óculos, eu não tinha forças para nada... eu chegava em casa e tava ficando só deitada e tava entrando em depressão, eu não conseguia mais sair, tem dias que eu varro a casa e eu limpo, mas daí amanhã eu não consigo sair do lugar, de tão fraca que eu fico, eu baquiei com isso, porque toda a vida eu andei muito, eu trabalhei muito, nunca tive parada [...]" Carla

"Não tenho vontade de levantar, não to com muita disposição pra sair pra rua... tem hora que eu é que fico meio revoltado... eu acho que eu não volto mais a fazer esse tratamento... eu vou voltar a trabalhar e fazer as minhas coisas [...]" Nelson

"[...] quando eu tava com aquela sonda eu nem saia mais porque tinha vergonha né, de sair com aquele trem no nariz [...]" Marcos

\section{DISCUSSÃO}

Em relação à caracterização sociodemográfica, constatou-se o predomínio do sexo masculino, o que coaduna com os achados na literatura (Souza et al., 2018). Esse resultado pode ser pelo fato do homem se expor mais aos fatores de risco, como fumo e ingestão de bebidas alcoólicas, bem como pode estar relacionado ao fato deles terem um menor autocuidado, realizando menos ações de prevenção e promoção de saúde (Carneiro et al., 2019).

Esse resultado aponta para um dos importantes dificultadores para 0 engajamento ao autocuidado, quer seja pela aceitação do homem à doença e ao tratamento como também pelo sentimento de invalidez e pela perda da identidade masculina. $\mathrm{E}$ traz contribuições aos profissionais de saúde para a implementação de ações mais efetivas de prevenção e de detecção precoce de doenças.

A média de idade dos participantes de 58,7 anos contraria ao encontrado na literatura, uma vez que há uma maior frequência do câncer em pessoas na terceira idade. Espera-se que o acometimento da 
doença se dê durante a senilidade, em decorrência de suas rotinas, hábitos $e$ crenças. $O$ fato de serem mais jovens pode por um lado favorecer 0 autocuidado pelo fato de as pessoas mais jovens terem preservado as habilidades e os processos mentais. Por outro lado, os efeitos da doença e do tratamento podem trazer impactos negativos para o autocuidado (Souza et al., 2018).

O contexto socioeconômico caracterizado por uma renda mensal de cerca de um salário mínimo e meio. os fatores ambientais relacionados ao fato de residir em município distante do centro de referência em oncologia, provocaram impactos negativos no autocuidado/cuidado. Este contexto impõe aos participantes a necessidade de contar com o transporte do sistema público de saúde que muitas vezes, não está disponível para 0 atendimento das necessidades do participante em casos de remoção e/ou obstrução do dispositivo. Essas dificuldades levaram os participantes a pagar por um profissional para a resolução dos problemas ou mesmo por adotar atitudes que podem incorrer em complicações. Silva, et al. (2018) apontaram em seu estudo o impacto das dificuldades financeiras no tratamento adequado de pessoas com câncer. A escassez de insumos necessários fornecidos pelo Sistema Único de Saúde SUS, obriga os participantes a adquiri-los com recursos próprios, e na falta deles, motivam condutas muitas vezes inapropriadas, como a reutilização do dispositivo, a reintrodução do dispositivo por pessoas não capacitadas, 0 uso de alimentos inapropriados nos dispositivos.

Os hábitos não saudáveis identificados no estudo, como o uso do tabaco e o consumo do álcool, podem comprometer ainda mais a saúde, uma vez que são fatores de risco para o desenvolvimento de cânceres de cabeça e pescoço, como encontrado em um estudo (Primo et al. 2016). As ações de educação em saúde desenvolvida por uma equipe multiprofissional fundamentada no trabalho multidisciplinar, no vínculo e na relação dialógica, podem tornar-se mais efetivas e contextualizadas de forma a estimular e engajar as pessoas em direção a hábitos de vida saudáveis (Barreto et al., 2019).

A presença do câncer de cabeça, pescoço e trato gastrointestinal, como identificados no estudo, pode levar as pessoas ao quadro de desnutrição e anorexia, devido à disfagia. A TNE se torna uma alternativa imprescindivel para suprir a alimentação e os requisitos para o alcance do autocuidado relacionados aos requisitos universais para a manutenção da estrutura e do funcionamento do ser humano em decorrência do desvio da saúde, provocado pelo câncer e seu tratamento. A TNE foi também a alternativa encontrada em estudo sobre o controle da nutrição enteral em pacientes em tratamento oncológico, com predominância as neoplasias malignas de cabeça e pescoço, e que apresentam risco/déficit nutricional (Lee et al., 2017).

A TNE é essencial nestes pacientes com 0 intuito de melhorar a qualidade de vida, manter ou recuperar a perda de massa magra, melhorar a cicatrização e fornecer energia, sendo implementada devido à anorexia, disfagia, aftas, mucosites e desnutrição severas, para melhorar 0 tratamento, evitar infecções e quadros de desnutrição (Souza et al., 2018).

Os resultados deste estudo apontaram para o predomínio da SNE por um tempo longo de permanência, seguido pela gastrostomia e jejunostomia. $O$ uso por tempo prolongado da TNE por meio da SNE, não é recomendado, devido aos riscos e complicações, como a broncoaspiração (Soria et al., 2017; Weisshmer \& Rech, 2017) e assim, concorre para diminuir a qualidade de vida dessas pessoas. Quando a TNE for recomendada por longos períodos deve-se optar pela gastrostomia ou pela jejunostomia (Ehrsson et al., 2015). 
A compreensão da pessoa quanto ao uso do dispositivo tem favorecido a sua adaptação e a adesão, e muitas vezes, se dá pela esperança de retomar a alimentação via oral (Cai et al., 2018; Teston et al., 2018 Halliday et al., 2017), o que pode favorecer 0 autocuidado/cuidado mediante a adaptação no cotidiano, como caracterizado no depoimento de Carla "[...] passava água, passava duas seringas, eu passava antes, pra ver se não tava entupido, e passava depois pra acabar de empurrar o remédio [...]".

O processo do autocuidado/cuidado torna-se intrínseco devido às experiências e a formação social, adquiridas no desenvolvimento, por meio da relação com pessoas mais experientes, e assim, elas criam estratégias que proporcionam 0 autocuidado (Rueda, 1999; Vitor, et al., 2010).

Percebeu-se que os participantes de modo geral, aprenderam as orientações dos profissionais de saúde, como relatado nos rituais para o preparo das medicações, para a maceração do comprimido, a sua diluição e administração, o que coloca em evidência a importância dessas orientações como facilitadoras do cuidado.

$O$ acesso aos insumos e aos serviços do SUS, as doações de ONGs ou de terceiros, influenciaram positivamente para 0 engajamento dos participantes ao autocuidado/cuidado. Esse resultado coaduna com os achados do estudo que constataram que 0 acesso aos serviços e insumos facilitaram o tratamento e o cuidado de pacientes oncológicos (Oliveira, et al., 2018).

Evidenciou-se a relevância da rede de apoio configurada pelos profissionais de saúde, pela família e pelos amigos, como propulsores no processo de adaptação e engajamento ao autocuidado/cuidado, 0 que possibilitou aos participantes um melhor enfrentamento do adoecimento e dos cuidados com o dispositivo. 0 apoio social assume relevância na medida que oferece ajuda emocional, financeira, instrumental e de relacionamento social (King et al., 2006) para minimizar as cargas decorrentes do câncer e do tratamento e para incentivar as pessoas com dispositivo de alimentação ao autocuidado.

A presença da família durante o processo de doença também exerce um papel importante para gerar segurança e amparo. Esses resultados corroboram com os achados de outros estudos (Primo et al., 2016; Teston et al., 2018; Tomaszewski, et al. 2017), tendo em vista que este apoio permite à pessoa se sentir-se amparada e com os vínculos mais fortalecidos, o que influencia positivamente no engajamento do autocuidado/cuidado.

Os profissionais de saúde devem mediante ao conhecimento das singularidades de cada pessoa, fornecer informações para que, a livre escolha da pessoa, tenha-se uma melhor adesão ao tratamento, aumentando assim, a responsabilidade pelo autocuidado/cuidado, uma vez que estimula a criar estratégias de enfrentamento, reduzir o estresse e alterar a percepção para os momentos de dificuldade (Oliveira, Stancato et al., 2018; Primo et al., 2016; Tossin et al., 2016).

Apesar do estigma existente frente ao adoecimento e a exclusão social, verificou-se pelos resultados que as pessoas se sentiram mais acolhidas por suas famílias, o que favoreceu aproximações por parte da pessoa e seus familiares. Compete ao profissional de saúde instigar tal aproximação, para que a pessoa possa ter uma rede de apoio facilitadora ao processo de reabilitação.

A autoaceitação como verificada em alguns depoimentos faz parte do processo de reabilitação e adaptação ao tratamento, em que as pessoas passam a se importar menos com os julgamentos e pensamentos dos outros, e levam a vida dentro da 
normalidade, sendo tal aspecto essencial para a sua reabilitação. Resultados de estudo (Cai et al., 2018), concluiu que com o passar do tempo há adaptação no uso diário do dispositivo, não sendo mais um problema o seu aspecto físico.

A busca pelo transcendente constitui-se uma força propulsora para o processo de doença, porque possibilitaram às pessoas força para 0 enfrentamento, para dar respostas as suas inquietações, para ressignificar o sofrimento, a fim de amenizar as suas preocupações acerca do futuro mediante a melhor aceitação de sua condição (Oliveira, Reis et al., 2018; Teston et al., 2018; e Tomaszewski et al., 2017).

Alguns fatores intrínsecos ou extrínsecos corroboraram para dificultar 0 autocuidado/cuidado, como as ações inapropriadas, que podem levar as complicações e prolongar o processo de reabilitação. Percebeu-se nos depoimentos a administração de alimentos em SNE por prescrição leiga, talvez por acreditarem que esses alimentos são fortes, 0 que proporcionariam às pessoas mais força para superar as adversidades do adoecimento. As dietas enterais são desenvolvidas com 0 intuito de sofrerem a absorção em locais específicos do trato gastrointestinal (Souza et al., 2018; Lee et al., 2017).

O conhecimento limitado sobre os cuidados com 0 dispositivo, com a administração da dieta e com o preparo e a administração dos medicamentos contribuiu para que alguns participantes adotassem atitudes que ocasionaram intercorrências, dentre as quais o aumento da infusão da dieta, a reutilização de dispositivos que podem favorecer a contaminação, falhas na limpeza da sonda antes e após administração de medicamentos, supressão da hidratação, dissolução dos medicamentos de forma inadequada, recolocação da sonda, obstrução da sonda e dobraduras na sonda.

0 manuseio inapropriado durante 0 preparo da dieta compromete a TNE, prolongando o período de permanência com o dispositivo, podendo ocasionar infecções. A exteriorização da sonda, considerada um evento adverso e falhas no gotejamento da dieta também foram encontrados em outros estudos (Farias, et al., 2011; Souza et al., 2018).

Nesse contexto, os profissionais de saúde precisam conhecer as apresentações dos medicamentos em sua forma líquida, tendo em vista que os relatos apontam que a grande maioria das medicações prescritas era de fármacos na sua apresentação sólida. Os resultados de um estudo (Farias et al., 2011) constataram uma melhora clínica em pacientes em uso dos dispositivos médicos de alimentação com medicações na sua apresentação líquida, já que alguns medicamentos podem perder o seu princípio ativo quando alterado a forma e apresentação original.

Outra questão importante é a necessidade de orientação dos profissionais de saúde sobre a associação dos medicamentos, uma vez que pode levar às interações medicamentosas, fazendo com que os mesmos percam a sua efetividade terapêutica. Os medicamentos devem ser administrados de forma separada, quando houver a necessidade de administração de mais um fármaco no mesmo horário (Farias et al., 2011). E ainda, a trituração de alguns medicamentos compromete a sua farmacocinética e farmacotécnica, modificando o local de ação inicial e gerando aglutinação de partículas que podem levar a obstrução do dispositivo, o que pode ser identificado nos depoimentos dos participantes do presente estudo (Gama et al., 2019; Joos et al., 2016).

Verificou-se no presente estudo 0 conhecimento deficiente sobre a hidratação antes e após a administração de medicamentos, o que corrobora para 0 aparecimento de complicações mecânicas como a obstrução do dispositivo e o déficit de autocuidado/cuidado. Recomenda-se a administração de $50 \mathrm{ml}$ de agua antes e após 
a administração de medicamentos (Silva, et al., 2018). As dificuldades de acesso dos participantes ao serviço em determinadas situações, pelo fato de algum deles residirem distante do município de origem do centro de referência em oncologia, e pela dependência do serviço público de saúde para o seu deslocamento. Essa barreira os levou a tomar atitudes inadequadas, tais como a reintrodução da sonda. Estudos de Oliveira, Reis et al. (2018), Oliveira, et al. (2018) também encontraram as dificuldades de deslocamento que alguns pacientes em tratamento oncológico apresentavam para comparecer ao serviço, 0 que gera desgastes físicos e psicológicos.

A dificuldade de acesso aos insumos, as medicações e as orientações são fatores preditivos para dificultar 0 autocuidado/ cuidado e comprometer a adesão ao tratamento. Tais dificuldades podem gerar consequências não apenas psicológicas e financeiras, como também postergar 0 processo de reabilitação e possível desmame do dispositivo (Cai et al., 2018; Oliveira et al., 2018b).

O processo de educação em saúde deve iniciar a partir da entrada da pessoa no serviço de saúde, haja vista que no momento da alta fornecer muitas orientações pode não ser devidamente apreendido, 0 que compromete a continuidade do cuidado no domicílio (Tossin et al., 2016).

Embora não tenha sido mencionado nos depoimentos dos participantes, vale acrescentar as orientações de Silva, et al. (2018) e Cheever e Hinkle (2015) frente aos cuidados que devem ser implementados nas rotinas da pessoa em uso de dispositivo médico de alimentação, como a elevação da cabeceira durante a administração da dieta, a higiene oral três vezes ao dia, os cuidados no local de inserção do dispositivo que envolve a inspeção e a limpeza, além da avaliação do volume residual para verificar estase. Tal conduta assume sua relevância, visto que, a depender da quantidade do volume residual, é necessário suspender a dieta, para evitar a distensão, a regurgitação e a broncoaspiração.

O processo do adoecimento e o uso do dispositivo geram uma série de transformações relacionadas aos aspectos emocionais, ao estigma, a autoimagem, a autoestima com importantes repercussões na identidade (Cai et al., 2018; Ehrsson et al., 2015). As pessoas passam por mudanças nas atividades de vida diária e nas relações sociais favorecendo o seu isolamento social, caracterizado pelas falas dos participantes. Esses resultados são similares aos encontrados nos estudos de Oliveira, et al. (2018) e Tomaszewski et al. (2017) que retrataram 0 quanto as rotinas diárias sofreram influências frente ao processo de adoecimento, o que pode levar a pessoa à inatividade e a perda de sua funcionalidade social.

As limitações físicas decorrentes da doença exigem modificações nas atividades de vida diária que podem constituir um fator desencadeador para a depressão, como relatado por Carla "[...] eu não tinha forças para nada... eu chegava em casa e tava ficando só deitada e tava entrando em depressão, eu não conseguia mais sair [...]". Resultados similares foram encontrados em estudo de Tomaszewski et al. (2017), que constataram a presença da impotência da pessoa mediante 0 adoecimento.

As ações devem ser desenvolvidas para melhorar não apenas a dimensão física como também as demais dimensões que compõem a integralidade da pessoa adoecida assim como da sua família, para que haja uma melhor adaptação ao tratamento, fazendo com que o dispositivo não seja considerado como um dificultador para as atividades diária. Para tanto, a pessoa precisa de uma rede de apoio, sejam esses familiares, amigos e/ou os profissionais, que possam auxiliar a pessoa no processo de doença e de reabilitação.

As representações simbólicas negativas que permeiam 0 imaginário da pessoa com 
câncer prejudicam a sua capacidade de lidar, de forma menos sofrida, com a doença e as suas consequências, e pode dificultar seu enfrentamento mais positivo da situação (Schulz et al., 2010). Esses sentimentos negativos afetam também toda a família, num âmbito físico, psíquico e social. Esses sentimentos denotam a estigmatização das pessoas em uso de sonda enteral, atrelado a sentimentos de tristeza e impotência. Assim, as pessoas tendem a sentir inúteis, 0 que favorece ainda mais o isolamento social e 0 afastamento de suas atividades anteriores à doença Teston et al. (2018). Tais sentimentos podem levar a pessoa a uma morte social e ao fracasso no tratamento (Primo et al., 2016; e Tomaszewski et al., 2016).

Para minimizar esses sentimentos e favorecer 0 processo de adaptação e reabilitação, torna-se fundamental 0 processo de comunicação sobre o processo de tratamento, suas possíveis consequências e prognósticos, evitando-se a idealização da pessoa frente ao seu estado de doença. Cabe aos profissionais um comprometimento ético e humanizado com a pessoa adoecida, o que favorece sua participação no processo de autocuidado/cuidado.

Assim, o enfermeiro é o profissional com competências para identificar as incapacidades que possam dificultar a implementação do autocuidado/cuidado. Nessa lógica, esse profissional, assim como toda a equipe de saúde, deve buscar pelo conhecimento nas melhores evidências científicas para estabelecer a comunicação terapêutica eficaz de modo a levantar as dificuldades das pessoas para que o manejo com o dispositivo seja realizado de forma mais assertiva possivel, para a continuidade do cuidado, evitando assim, as suas intercorrências (Dengo et al., 2016).

\section{Conclusões}

Os dados permitem concluir que a neoplasia maligna, um desvio de saúde e seu tratamento afetam o requisito universal para o autocuidado, a alimentação. Para suprir essa necessidade, as pessoas são submetidas ao uso de dispositivos enterais, gástricos ou no jejuno e precisam aprender a lidar com eles para a efetivação do autocuidado/cuidado.

Os facilitadores do autocuidado/cuidado referem-se ao aprendizado que eles decodificaram das orientações dos profissionais de saúde, ao acesso aos insumos e medicações, ao apoio da família, dos amigos, das ONGs e dos profissionais de saúde e da fé que potencializa a sua espiritualidade.

Por outro lado, os dificultadores que contribuíram para 0 déficit de autocuidado/cuidado foram 0 manejo inadequado, muitas vezes pela falta de orientações compreensíveis, a dificuldade de acesso aos insumos/medicações e orientações, o contexto econômico, os fatores ambientais $e$ as repercussões negativas da doença e do dispositivo que comprometeram não apenas a autoimagem, mas as relações sociais e a sua identidade.

O processo de educação em saúde com vistas ao engajamento da pessoa com dispositivo de alimentação ao cuidado e autocuidado é complexo, uma vez que exige dos profissionais de saúde adequada comunicação, experiência, conhecimento fundamentado nas melhores evidências, competência e conhecimento do contexto sociocultural da pessoa sob os seus cuidados. A esses profissionais, principalmente ao enfermeiro, competem planejar e implementar ações de educação em saúde, numa perspectiva da integralidade, da clínica ampliada e da relação dialógica para a partilha de orientações.

A relevância desse estudo contribui para fomentar reflexões quanto ao cuidado ofertado pela enfermagem, qualificando-a para um melhor atendimento $e$ reconhecimento das necessidades dessas 
pessoas, afim de que o processo de transição hospital-domicílio possa ser realizado como um continum de cuidado, 0 que contribui para uma melhor convivência com o dispositivo e para reorientar a sua práxis. E ainda, para reconhecer que cada pessoa tem um modo especial de aprendizagem e todos tem o potencial para 0 autocuidado/cuidado. É preciso considerar o contexto de vida e as singularidades das pessoas no processo de educação em saúde, 0 que aponta como importante contribuição desse estudo.

Dentre as limitações do presente estudo destaca-se o número de participantes, tendo em vista a desatualização do cadastro. Torna-se importante o desenvolvimento de outros estudos, que possam envolver um número maior de participantes e suas diferentes vivências com os dispositivos de alimentação.

\section{REFERÊNCIAS}

Barreto, A.C.O., Rebouças, C.B.A, Aguiar, M.I.F., Barbosa, R.B., Rocha, S.R., Cordeiro, Melo, K.M., \& Freitas, W.J.F.F. (2019). Perception of the Primary Care multiprofessional team on health education. Revista Brasileira de Enfermagem, 72, 266-73. https://doi.org/10.1590/0034-7167-2017$\underline{0702}$

Braun, V. \& Clarke, V. (2006). Using thematic analysis in psychology. Qualitative Research in Psychology, 3(2), 77101. https://doi.org/10.1191/1478088706 qp0630a

Cai, Q., Li, F., \& Zhou, Y. (2018). Experiences of Chinese patients with Crohn's disease in the self-administration of nasogastric feeding: A descriptive qualitative study. PLOS ONE,1(7), 1-18.

https://doi.org/10.1371/journal.pone.0201 421

Cassel, J. (1976). The contribution of the social environment to host resistance. American Journal of Epidemiology, 104(2), 107-123. https://doi.org/10.1093/oxfordjournals.aje. $\underline{\text { a112281 }}$
Castel, R. (1995). As Metamorfoses da Questão Social. Petrópolis. Editora Vozes.

Carneiro, V. S. M., Adjuto, R. N. P. \& Alves, K. A. P. (2019). Saúde do homem: identificação e análise dos fatores relacionados à procura, ou não, dos serviços de atenção primária. Arquivos de Ciências da Saúde da UNIPAR, 23 (1), 35-40.

https://doi.org/10.25110/arqsaude.v23i1. 2019.6521

Cheever, K.H. \& Hinkle, J.L. (2015). Manual de enfermagem médico-cirúrgica. Editora Guanabara Koogan.

Dengo, D. C., Benincá, S. C., Mazur, C. E. \& Valduga, L. (2016). Terapia nutricional domiciliar: perfil nutricional dos usuários e qualidade microbiológica na preparação das fórmulas. Revista UNINGÁ Review, 25(3), 18-24.

http://34.233.57.254/index.php/uningarevi ews/article/view/1775

Ehrsson, Y. T., Sundberg, K., Laurell, G. \& Eklof, A. L. (2015). Head and neck cancer patients' perceptions of quality of life and how iti $s$ affected by the disease and enteral tube feeding during treatment. Upsala Journal of Medical Sciences, 120(4), 280-289.

https://doi.org/10.3109/03009734.2015.1 075630

Farias, M. A., Didonet, J., Predebon, S. \& Schwarzbold, C. V. (2011). Estruturação de orientação farmacêutica para com medicamentos por sonda nasoenteral: um estudo de caso. Revista Brasileira de Farmácia, 92(4), 378-383.

http://www.rbfarma.org.br/files/rbf-201192-4-23-378-383.pdf

Fialho, A. V. M., Pagliuca, L. M. F., \& Soares, E. (2002). Adequação da teoria do déficit de teoria do déficit de autocuidado no cuidado domiciliar à luz do modelo de Barnum. Revista Latino-Americana de Enfermagem, 10(5), 715-720. https://doi.org/10.1590/S010411692002000500014

Foster, P.C. \& Bennett, A. M. (2000). Dorothea E. Orem. In: George, J. B. et al. (Org) Teorias de enfermagem: os fundamentos à prática profissional. (pp. 83-89). Porto Alegre: Artes Médicas Sul Ltda. 
Gama, F. L.S., Leão, N.M.L., Xavier, M.P., Sousa, S.F., Vale, B.N., Santana, V.L. (2019). Elaboração de protocolos para administração de medicamentos sólidos orais por sondas de nutrição enteral. Revista Amazônia: Science \& Health, 7(1), 26-49.

http://ojs.unirg.edu.br/index.php/2/article/ view/2760/pdf

Halliday, V., Baker, M., Thomas, A. L. \& Bowrey, D. (2017). Patient and family caregivers' experiences of living with a jejunostomy feeding tube after surgery for esophagogastric cancer. Journal of Parenteral and Enteral Nutrition, 41(5), 837-843.

https://doi.org/10.1177/01486071156041 $\underline{14}$

Instituto Nacional do Câncer (2020, 26 de Abril). Estimativa 2018: Incidência de Câncer no Brasil. 2018. https://www.inca.gov.br/estimativa/estado -capital/minas-gerais-belo-horizonte

Joss, E., Mehuys, E., Van Bocxlaer, J., Remon, J.P., Van Winckel, M. \& Boussery, K. (2016). Knowledge of staff members of residential care facilities for individuals with intellectual disability on medication administration via enteral feeding tube. Journal of Intellectual Disability Research, 60(11), 1066-1072.

https://doi.org/10.1111/iir.12263

King, G., Willoughy, C., Specht, J.A. \& Brown, E. (2006). Social support processes and the adaptation of individuals with chronic disailities. Qualitative Health Research, 16, 902-925.

https://doi.org/10.1177/10497323062899 $\underline{20}$

Lee, A., Oliveira Filho, R.S., Cardenas, T.C., Ozório, G.A., Gropp, J.P.L. \& Waitzberg, D.L. (2017). Quality control of enteral nutrition therapy in cancer patients at nutritional risk. Revista Nutrición Hospitalaria, 34(2), 264-270. https://doi.org/10.20960/nh.721

Mitchell, S., Williams, J.P., Bhatti, H., Kachaamy, T., Weber, J. \& Weiss, G.J. (2017). A retrospective matched cohort study evaluating the effects of percutaneous endoscopic gastrostomy feeding tubes on nutritional status and survival in patients with advanced gastroesophageal malignancies undergoing systemic anti-cancer therapy. PLOS ONE, 12(11), 1-8.

https://doi.org/10.1371/journal.pone.0188 $\underline{628}$

Nascimento, S. G., Carvalho, C. P. A. L., Silva, R. S., Oliveira, C. M. \& Bonfim, C. V. (2018). Decline of mortality from cervical cancer. Revista Brasileira de Enfermagem, 71(1), 585-590.

http://dx.doi.org/10.1590/0034-71672016-0530

Oliveira, A. M., Stancato, K. \& Silva, E. M. (2018). Formação do enfermeiro: políticas públicas na atenção oncológica. Revista Enfermagem em Foco, 9(3), 48-53.

https://doi.org/10.21675/2357707X.2018.v9.n3.1175

Oliveira, J. M., Reis, J. B. \& Silva, R. A. (2018). Busca por cuidado oncológico: percepção de pacientes e familiares. Revista de Enfermagem UFPE online, 12(4), 938946.

https://periodicos.ufpe.br/revistas/revista enfermagem/article/view/231359/28643

Primo, C. C., Cesar, F. D., Lima, E. F. A., Caniçali, R. A. \& Leite, F. M. C. (2016). Assistência de enfermagem a pacientes com câncer de cabeça e pescoço submetidos à radioterapia. Revista de Pesquisa: Cuidado é Fundamental Online, 8(1), 3820-3831.

http://www.seer.unirio.br/index.php/cuida dofundamental/article/view/4173/pdf_179 $\underline{6}$

Rueda, S. (1999). Dorothea E. Orem. Aproximación a su teoría. Revista ROL de Enfermería, 22(4), 3019-311.

https://www.erol.es/articulospub/articulospub_paso3.p hp?articulospubrevista=22(4)\&itemrevist $\mathrm{a}=309-311$

Sales, C.A. \& Molina, M.A.S. (2004). O significado do câncer no cotidiano de mulheres em tratamento quimioterápico. Revista Brasilera de Enfermagen, 57(6), 720-723.

http://dx.doi.org/10.1590/S003471672004000600018

Silva, D. K. V., Pessoa, E. T. \& Veloso, H. H. (2018). Dificuldades Financeiras e seus Impactos no Tratamento de Pacientes com Câncer: Uma Realidade Vivenciada 
no Projeto Ero. Revista Saúde e Ciência Online, 7(2), 157-169.

http://dx.doi.org/10.35572\%2Frsc.v7i2.62 6

Silva, T. P., Ribeiro, C. R. G., Resck, Z. M. R., Fava, S. M. C. L. \& Dázio, E. M. R. (2018). Cuidado de enfermagem à pessoa com gastrostomia: revisão integrativa. Revista Estima, 16, 1-7.

http://dx.doi.org/10.30886/estima.v16.374

Schulz, V. L., Menezes, N. N. T. \& Peres, R. S. (2010). Pesquisa em psicooncologia no Brasil: desenvolvimentos recentes sobre aspectos psicológicos do câncer de mama. Clinica e Instituição, 5, 59-70.

Soria, A., Santacruz, E., Vega-Piñeiro, B., Gión, M., Molina, J., Villamayor, M., Mateo, R., Riveiro, J., Nattero, L. \& Botella-Carretero, J.I. (2017). Gastrostomy vs nasogastric tube feeding in patients with head and neck cancer during radiotherapy alone or combined chemoradiotherapy. Revista Nutrición Hospitalaria, 34(3), 512-516.

http://dx.doi.org/10.20960/nh.680

Souza, I. A., Bortoletto, M.M., Dias, A.M.N., Almeida, N.M., Ribeiro, L.C. \& Mendonça, E.G. (2018). Nutrição enteral em pacientes oncológicos: diferenças entre 0 que é prescrito e administrado. Revista Nutrición Clínica y Dietética Hospitalaria, 38(2), 31-38.

https://doi.org/10.12873/382lury

Teston, E. F., Fukumori, E.F.C., Benedetti, G.M.S., Spigolon, D.N., Costa, M.A.R. \& Marcon, S.S. (2018). Sentimentos e dificuldades vivenciadas por pacientes oncológicos ao longo dos itinerários diagnóstico e terapêutico. Escola Anna Nery Revista de Enfermagem, 22(4), 1-8. http://dx.doi.org/10.1590/2177-9465-ean2018-0017

Tomaszewski, A. S., Oliveira, S. G., Arrieira, I. C. O., Cardoso, D. H. \& Sartor, S. F. (2017). Manifestações e necessidades referentes ao processo de morte e morrer: perspectiva da pessoa com câncer. Revista de Pesquisa: Cuidado é Fundamental Online, 9(3), 705-716. https://doi.org/10.9789/2175-

5361.2017.v9i3.705-716

Tossin, B. R., Souto, V.T.,Terra, M.G., Siqueira, D.F.,Melo, A.L. \& Silva, A.A. (2016). As práticas educativas e 0 autocuidado: evidências na produção científica da enfermagem. Revista Mineira de Enfermagem, 20, 1-8.

http://www.dx.doi.org/10.5935/14152762.20160010

Vitor, A. F., Lopes, M. V. O. \& Araújo, T. L. (2010). Teoria do déficit de autocuidado: análise da sua importância e aplicabilidade na prática de enfermagem. Escola Anna Nery Revista de Enfermagem, 14(3), 611-616. http://dx.doi.org/10.1590/S141481452010000300025

Waitzeberg, D. L. Almeida, D.F., Alves, C.C., Campos, L.N., Cardenas, T.C., Lima, S.C.T.C., Silva, L.C. \& Wilson, D.B.C. (2013) Nutrição e câncer. Em: HOFF, P. M. G. et al. Tratado de Oncologia. (pp. 1321-1354). Editora Atheneu.

Weissheimer, A. \& Rech, C. R. A. (2017). 0 papel da terapia nutricional nos tumores de cabeça e pescoço. Revista de Nutrição e Vigilância em Saúde, 4(1), 80-86. http://www.revistanutrivisa.com.br/wpcontent/uploads/2018/04/nutrivisa-vol-4num-1-10.pdf

Todos los trabajos publicados en Revista Salud \& Sociedad (ISSNe:0718-7475) están sujetos a una licencia Creative Commons Reconocimiento 4.0 Internacional

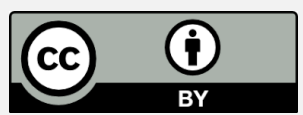




\section{RESUMO}

INTRODUÇÃO: 0 câncer e seu tratamento podem afetar a capacidade de alimentação, necessitando da terapia nutricional enteral, a qual demanda um autocuidado e cuidado apropriados. OBJETIVO: conhecer 0 autocuidado/cuidado de pessoas com câncer em uso de dispositivos médicos de alimentação num município do Estado de Minas Gerais. MÉTODO: investigação qualitativa obtida por meio de entrevistas gravadas, utilizando um instrumento semiestruturado, com pessoas atendidas num centro de referência em oncologia. RESULTADOS: construiu o tema central requisitos de autocuidado/cuidado universal por desvio de saúde: forças propulsoras e restritivas e como subtemas facilitadores para 0 engajamento ao autocuidado/cuidado e dificultadores para 0 engajamento ao autocuidado/cuidado. DISCUSSÃ0: Os resultados contribuem para a práxis dos profissionais de saúde, principalmente do enfermeiro para repensar a relevância das orientações no processo de transição hospital-domicílio e para reconhecer que cada pessoa tem um modo especial de aprendizagem e todos têm o potencial para o cuidado/autocuidado.

PALAVRAS-CHAVE: Autocuidado, Nutrição enteral, Oncologia, Enfermagem.

\section{RESUMEN}

ANTECEDENTES: El cáncer y su tratamiento pueden afectar la capacidad de la alimentación, necesitando de terapia nutricional entérica, que exigen, autocuidado y cuidado apropiados a cada caso. OBJETIVO: conocer el autocuidado/cuidado de personas con cáncer en uso de dispositivos de alimentación médica en un municipio del estado de Minas Gerais. MÉTODO: investigación cualitativa a través de entrevistas grabadas, usando un instrumento semiestructurado, con personas atendidas en un centro de referencia en oncología. RESULTADOS: los requisitos allí construyeron el sujeto central del autocuidado/cuidado universal para el desvío de la salud: fuerzas propulsoras y restrictivas y como subtemas de facilitación para el compromiso de autocuidado/cuidado y dificultadores para el compromiso de autocuidado/cuidado. Consideraciones finales: los resultados contribuyen a la praxis de los profesionales de salud, principalmente de la enfermería observando atentamente la importancia de las orientaciones al paciente en el proceso de transición hospital-domicilio y reconocer que cada persona tiene su forma de aprendizaje diferenciada y que, todos tienen los pacientes tienen el potencial para el autocuidado/cuidado.

PALABRAS CLAVE: Autocuidado, Nutrición enteral, Oncología médica, Enfermeria. 